Tesis. Año 13, 12(15), 2019, 43-62

\title{
La paradoja de Aquiles y la tortuga como una falacia del continuo
}

\author{
Rafael Félix Mora Ramírez \\ rafael.mora@unmsm.edu.pe
}

\section{Resumen}

En este trabajo analizo la paradoja de Aquiles y la tortuga. Para ello, comienzo por la definición de 'falacia' y 'paradoja' y, además, expongo la clasificación de las paradojas establecida por Quine. Enseguida, presento la paradoja de Zenón y luego muestro una manera de resolverla usando matemática básica. Esta solución permite sostener que la supuesta paradoja solo era una falacia. En la parte final del presente artículo muestro que la mencionada paradoja era un caso muy especial de la falacia de la continuidad.

Palabras clave: Pseudoparadoja, "Aquiles", falacia del Continuo, clasificación de las paradojas de Quine.

\section{Abstract}

In this paper analyzed the paradox of Achilles and the tortoise. To do this, start with the definition of 'fallacy' and 'paradox' and, in addition, I am putting the classification of the paradoxes established by Quine Immediately, presented the paradox of Zeno and then a way to resolve it using basic math. This solution allows me argue that the alleged paradox only was a fallacy. In the final part of this article, I show this paradox was a very special case of the fallacy of the continuity.

Key words: Pseudoparadox, "Achilles”, fallacy of continuous, classification of the paradoxes of Quine. 


\section{La paradoja de Aquiles y la tortuga como una falacia del continuo}

\section{Falacia}

Falacia deriva del latín fallacia-ae, que quiere decir engaño, fraude, superchería, mentira con la que se intenta dañar a alguien. En efecto, las falacias nos engañan, haciéndonos admitir como válidos razonamientos que no lo son. En definitiva, las falacias son razonamientos psicológicamente persuasivos, pero lógicamente defectuosos. A pesar de que un simple error visible sería detectado de inmediato, sin embargo, estos logran convencer a la audiencia a la que están dirigidos porque sus errores se hallan revestidos de una apariencia de corrección.

En el lenguaje coloquial, el término falacia se emplea a menudo con poco rigor para designar cualquier idea equivocada, creencia falsa o conocimiento erróneo. Enseguida, mostramos algunos ejemplos:

1) Todos los judíos son avaros.

2) Ninguna mujer es infiel.

3) Todos los africanos son negros.

4) Todo miraflorino es pudiente.

5) Todos los hombres son iguales.

6) Todos los argentinos son soberbios.

7) Todos los homosexuales tienen sida.

8) Ningún obrero es capitalista.

9) Todas las aves pueden volar.

10) Todos los políticos son corruptos.

Estas instancias serán llamadas expresiones falaces porque aluden a proposiciones falsas. Pero esto es también un uso impropio. Por ejemplo, si alguien dice que "Paolo se escapó de su colegio" cuando en realidad Paolo estuvo en su 
colegio, entonces Paolo podrá acusarlo de estar diciendo falacias porque está expresando falsedades sobre él.

En un sentido más estricto o más técnico, los lógicos usan el término falacia como denotando algún error en un razonamiento. De ahí que para que haya falacia es menester que haya algún razonamiento, aunque sea en el sentido de "aparente".

Una falacia es un tipo de razonamiento incorrecto. Pero no todo razonamiento incorrecto es una falacia. Algunos razonamientos son tan obviamente incorrectos que no engañan a nadie. Por ejemplo:

P.1) Algunos jueces son honrados.

C) Por lo tanto, todos los jueces son honrados

es un razonamiento incorrecto, pero no es una falacia.

En lógica se acostumbra reservar el nombre de falacia a aquellos razonamientos que, aunque lógicamente incorrectos, son psicológicamente persuasivos. Por ello, una falacia es un tipo de razonamiento incorrecto que se presenta como si fuera correcto, pero resulta no serlo cuando se lo analiza cuidadosamente. Por ejemplo:

P.1) Las sociedades están compuestas de individuos

P.2) Los objetivos de los individuos son siempre, en última instancia, egoístas.

C) Por lo tanto, ninguna sociedad puede tener objetivos altruistas

P.1) Mario ha estado involucrado en casos de corrupción

C) Por ende, la propuesta de Mario para controlar la violencia en el fútbol no es la mejor

P.1) Los seres humanos gritan y sienten dolor cuando se los golpea.

P.2) Los animales gritan cuando se los golpea.

C) En conclusión, los animales sienten dolor cuando se los golpea

En el primer caso, se comete la falacia de creer que las propiedades de los componentes de las partes determinan una propiedad de todo un compuesto (falacia de composición). En el segundo caso, la falacia estriba en desacreditar la propuesta de Mario solo porque él ha estado involucrado en la corrupción (falacia ad hominem). En este caso, aunque sea cierto que Mario haya colaborado (consciente o inconscientemente, directa o indirectamente) con la degeneración de la política de su país, su propuesta puede ser tomada en cuenta si es que logra 
frenar las incidencias de violencia en las calles de la ciudad. En el tercer caso, se busca asociar mediante una analogía que lo que le afecta al ser humano también le afecta al animal (falacia de causa falsa).

Todas estas ejemplificaciones representan falacias que buscan derivar una conclusión sin respetar la relación de consecuencia lógica entre las premisas y la conclusión. En palabras de García Damborenea:

A todas las formas de argumentación que encierran errores o persiguen fines espurios, los llamamos falacias. El término procede del latín fallacia, que significa engaño, y lo empleamos como sinónimo de sofisma, palabra que acuñaron los griegos para designar el argumento engañoso.

Ya se ve que la terminología es imprecisa porque mezcla errores de razonamiento (...), con maniobras extra-argumentales (...), e incluye también los falsos argumentos que se emplean con la intención de engañar o desviar la atención (...). Todos tienen una cosa en común: adoptan la apariencia de un argumento e inducen a aceptar una proposición que no está debidamente justificada. Unas veces nos engaña nuestro juicio y otras las mañas de nuestro interlocutor. (2000, p. 123)

En otras palabras, un buen argumento, por lo general, establece la verdad de su conclusión. Si no lo logra, pero aun así se expone ante el público oyente para convencerlo, se convertirá en una falacia. Este mal argumento puede fallar de dos maneras:

(...) La primera es suponer alguna proposición falsa como una de las premisas del argumento (...)

La segunda forma en que el argumento puede fracasar (...) es que sus premisas no (...) impliquen [la conclusión]. Aquí nos hallaremos en la región específica del lógico, cuyo interés principal es el de las relaciones lógicas entre las premisas y la conclusión. Un argumento cuyas premisas no implican su conclusión es un argumento cuya conclusión puede ser falsa aun si todas sus premisas fuesen verdaderas. (Copi y Cohen, 2001: 125-126)

\section{Paradoja}

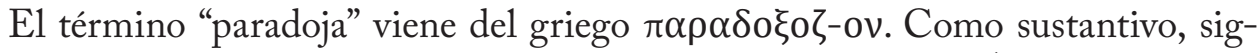
nifica etimológicamente: más allá, o "contrario a la opinión” (Blánquez, 1985, p. 1097), o a lo convencionalmente aceptado. Asimismo, siguiendo a Ferrater Mora (1994, p. 2693), la paradoja es " un acontecimiento que parece asombroso que pueda ser tal como se dice que es". Como adjetivo, la palabra paradójico (a) funciona como sinónima de "inesperado, increíble, extraño, maravilloso, raro, singular y extraordinario” (Pabón, 1997, p. 450). 
Coloquialmente, el término "paradoja" designa a aquella sentencia inverosímil, increíble y/o contradictoria que va en contra de lo común, que es opuesta a lo que se cree, que se opone a lo que se toma por verdadero. Así, las paradojas, en tanto sentencias, son expresiones breves que encierran contradicción o generan sorpresa por lo absurdo de su contenido.

1) Todos somos iguales, pero unos más iguales que otros.

2) Si quieres paz, prepárate para la guerra.

3) No hay mal que por bien no venga.

4) El hombre es como el oso mientras más feo, más hermoso.

5) Me da pena ser tan cruel.

6) Es de mala suerte ser supersticioso.

7) Está prohibido prohibir.

8) Soy ateo gracias a Dios.

9) Toda regla tiene excepciones.

10) En mi debilidad está mi fortaleza.

En sentido técnico, las paradojas son tipos especiales de contradicción, aquella dada por una oración cuya verdad implica su falsedad, del mismo modo que su falsedad implica su verdad. Por este singular rasgo es que es plausible señalar que la paradoja asombra, toda vez que nos enfrenta a la formulación de un enunciado absurdo, en relación con el cual extrañamente parece no ser posible señalar la causa que explique por qué adopta dicha forma. Esta definición funciona para el caso de la paradoja de El Mentiroso. Examinemos dicha paradoja: supongamos que existe una oración que afirma de sí misma que es falsa.

Hipótesis:

(1) La oración (1) es falsa.

Problema: ¿es verdadera o es falsa la oración (1)?

Por un lado, si (1) es verdadera, entonces se cumple lo que dice. Pero, si lo que dice se cumple, entonces (1) es falsa. Es decir, si (1) es verdadera, entonces (1) es falsa.

Por otro lado, si (1) es falsa, entonces lo que dice dicha oración se confirmaría. Pero, si se confirma lo dicho por esa oración, entonces (1) es verdadera. Es decir, si (1) es falsa, entonces (1) es verdadera.

Hay que decir que, en contraposición con el sentido coloquial de la paradoja que la define como la sentencia inverosímil, increíble y contradictoria que va en contra de lo común, al definir técnicamente una paradoja como aquel ente lingüístico cuya verdad implica su verdad y viceversa; se está considerando a la 
paradoja desde el aspecto del proceso racional que la genera. Así, es el razonamiento, como aquel proceso de la inferencia que pone en juego reglas lógicas, el elemento principal que permite identificar a la paradoja. En esta línea de pensamiento, Jonathan Vogel define a la paradoja sosteniendo que es un argumento de premisas aceptables, pero con una conclusión inaceptable (Dancy y Sosa, 1992, p-. 324). Esta será la definición de paradoja en sentido técnico que acogeremos. Veamos un caso para notar cómo se aplica esta definición. Por ejemplo, las premisas aceptables de la paradoja de El Mentiroso serían:

1) Todo enunciado que tiene significado es verdadero o falso.

2) La oración que dice de sí misma que es falsa tiene significado. y, la conclusión inaceptable sería:

C) Si la oración que dice de sí misma que es falsa, es verdadera entonces dicha oración es falsa, y si dicha oración es falsa, entonces ella misma sería verdadera.

Michael Clark en "El gran libro de las paradojas" (2009) sostiene que, a decir de Mark Sainsbury, una paradoja es un argumento en el que una conclusión aparentemente inaceptable se deriva, mediante un razonamiento aparentemente aceptable, a partir de premisas también aparentemente aceptables. Algo semejante dice Roger Scruton (2003) cuando afirma que una paradoja es un argumento que conduce por etapas racionales a una contradicción a partir de premisas intuitivamente aceptables.

${ }^{1}$ También, según Alberto Clemente de la Torre en Física Cuántica para filósofos: "la palabra [paradoja] [implica] (...) llegar a una conclusión evidentemente falsa o absurda por un razonamiento aparentemente correcto" (2000: 98). Más específicamente, de acuerdo con Piotr Łukowski (2011), en una paradoja concurren tres cosas:

1.- Se han utilizado correctamente las reglas de la inferencia.

2.- Se ha formulado adecuadamente el razonamiento.

3.- Hay la certeza de que nuestras opiniones son, hasta el momento, racionales.

Y sucede que, a pesar de lo anterior, se llega a una contradicción. Por lo tanto, la paradoja es un argumento legalmente correcto que, sin embargo, deviene en una contradicción.

Así, por medio de la estructura de explicación que se suele usar para poder exponer el significado del término falacia ${ }^{2}$, podemos también proporcionar una manera de comprender al vocablo paradoja. Consideramos que esto es posible porque ambos tipos de conceptos son de índole lógica. Así pues, una primera nota que señalaremos es que comparten la singularidad de presentarse como 
tipos especiales de argumentos ${ }^{3}$. Mientras que las falacias son razonamientos psicológicamente persuasivos pero lógicamente defectuosos, las paradojas pueden definirse como razonamientos que son lógicamente impecables pero psicológicamente extraños porque, si bien en una paradoja se procede con rigor lógico, sin embargo, se llega a resultados que son: o bien, absurdos (según la creencia común); o bien, contradictorios (atentando así contra un principio lógico clásico). ${ }^{4}$ Asimismo, como segundo aspecto que tienen en común ambos conceptos podemos decir que mientras que la falacia puede ser explicada tanto en un sentido coloquial (creencia extendida aunque falsa) como en un sentido técnico (razonamiento que pretende persuadir sobre alguna cuestión y que oculta algún error); igualmente, una paradoja también se puede explicar en un sentido coloquial (sentencia inverosímil, increíble y/o contradictoria) y en un sentido técnico (argumento de premisas aceptables pero con una conclusión inaceptable). Continuando con las similitudes, en tercer lugar, sostenemos que mientras que la falacia suele estar asociada a los términos paralogismo y sofisma; la paradoja comúnmente se le vincula a los términos aporía y antinomia. Finalmente, la conocida clasificación de las falacias en: atingentes y ambiguas; puede ser vista como semejante a la clasificación de las paradojas en: semánticas y lógicas.

\section{Clasificación de las paradojas de Quine}

W. V. O. Quine en The Ways of Paradox (1976) en base a la definición técnica de paradoja que hemos proporcionado (es decir, un argumento de premisas aceptables, pero con una conclusión inaceptable) nos propone una división de las paradojas en: verídicas, falsídicas y antinomias; con el fin de distinguir algunos detalles que tienen varios casos específicos de paradojas.

Quine habla de 'paradojas verídicas' -en la cuales lo que se pretende establecer es verdadero- y de 'paradojas falsídicas' —en las cuales lo que se pretende establecer es falso-. Los términos 'verídico' y 'falsídico' provienen del latín veridicus y falsidicus y atribuyen verdad (realidad) o falsedad (ilusión) a los sustantivos que califican. Estos términos tienen cierta relación con el concepto de validez lógica.

Según Quine, aquellas paradojas en las que la presuposición de la existencia de ciertas entidades conduce a una contradicción serán denominadas paradojas verídicas, puesto que este tipo de paradojas se presentan cuando la afirmación que nos resulta inicialmente absurda, notamos luego que es verdadera, al comprender el razonamiento que la justifica. En algunos casos, la paradoja verídica se constituye como una reducción al absurdo que al tener la forma lógica: $\mathrm{P} \rightarrow$ $\left(\mathrm{Q}^{\wedge} \sim \mathrm{Q}\right)$ culmina en $\sim$ P. Revisemos el caso de la paradoja de Galileo.

En la paradoja de Galileo, planteada en sus Diálogos sobre dos nuevas ciencias, Galileo (1945, p. 57- 59), sostiene la aparente falta de consistencia en la 
relación entre números naturales y números cuadrados. Da la impresión de que hay más números enteros $(1,2,3, \ldots)$ que cuadrados de dichos números $(1,4$, $9, \ldots)$. Pero es posible emparejar los números enteros con sus cuadrados:

$\begin{array}{lllllllll}1 & 2 & 3 & 4 & 5 & 6 & 7 & 8 & \ldots \\ \downarrow & \downarrow & \downarrow & \downarrow & \downarrow & \downarrow & \downarrow & \downarrow & \\ 1 & 4 & 9 & 16 & 25 & 36 & 49 & 64 & \ldots\end{array}$

Sin embargo, si consideramos que los cuadrados son parte de los naturales y que el todo es siempre mayor que la parte, entonces podemos concluir que los naturales serán más que los cuadrados. No obstante, podemos darnos cuenta de que ambos tipos de número son infinitos (en la misma medida). Así, llegamos a la idea de que la parte es igual que el todo, lo cual es contradictorio con nuestro sentido común. Sin embargo, hay que notar que la relación entre estos dos conjuntos de números nada tiene de aparente o engañosa puesto que, en verdad, dichos conjuntos son igual de numerosos, es decir, tienen la misma cantidad de elementos. Esto último se puede probar utilizando algunas herramientas básicas de la teoría de conjuntos, específicamente, la relación de correspondencia biunívoca.

Otros ejemplos de paradojas verídicas están constituidos por la familia argumental de paradojas de Russell y estas son: la paradoja del barbero, la paradoja del alcalde y la del catálogo. Todas estas son versiones populares de la conocida paradoja de Russell.

Otras veces, nuestras sospechas están totalmente fundadas y esa alerta de que alguna mala información se ha dejado filtrar no debe ser descartada, sino que tenemos todo el derecho de iniciar una investigación que nos permita estar seguros de que teníamos razón al atribuirle toda la responsabilidad a tal o cual premisa o regla deductiva. Ya Quine bautizó esta última situación con el nombre de paradoja falsídica, puesto que se trata de un razonamiento que no solo aparenta ser falaz, sino que lo es. Notemos que este tipo de paradojas falsídicas se constituirán como argumentos incorrectos que, además, pretenderán tener la apariencia de ser válidos. En resumen, la paradoja falsídica es un argumento inválido del cual se concluyen solo cosas falsas (Ferrater Mora, 1994, p. 2693). Por ejemplo, en 1847, Augustus De Morgan publica su texto Lógica Formal o el cálculo de inferencia y demuestra que $2=1$. Supongamos que $\mathrm{x}=1$. Luego, multiplicando ambos miembros de la igualdad por $\mathrm{x}$, obtenemos: $\mathrm{x}^{2}=\mathrm{x}$. Ahora, restemos 1 a cada lado de la nueva igualdad: $\mathrm{x}^{2}-1=\mathrm{x}-1$. Enseguida, dividamos ambos miembros por $x-1$, y nos queda: $x+1=1$. Finalmente, como ya sabíamos que $\mathrm{x}=1$, solo reemplazamos $\mathrm{x}$ y así nos queda que $2=1$. Debemos considerar que aquí existe un claro engaño, pues no podemos dividir por $\mathrm{x}-1$, pues $\mathrm{x}-1=0$, y la división por cero no existe. A este tipo de paradojas en las que algo extraño se sostiene por una falsa demostración se le conoce como paradoja falsídica. 
Finalmente, tenemos a la antinomia. Esta presenta tal contradicción interna que, por un lado, tiene una conclusión inaceptable, pero, por el otro, somos incapaces de descubrir en dónde se halla el error. La presencia de antinomias nos obliga a cambiar nuestros esquemas conceptuales, principios lógicos y axiomas. Dentro de esta clasificación, la antinomia es la que representa un verdadero desafío al intelecto humano. Como ejemplos de antinomias podemos mencionar a las paradojas de El Mentiroso, de Grelling ${ }^{5}$ y de Russell ${ }^{6}$.

\section{Pseudoparadojas}

Hemos definido a la paradoja como aquel argumento que tiene premisas aceptables pero conclusión inaceptable. Ahora bien, aquellos argumentos paradójicos que simplemente sean argumentos inválidos (falaces) serán llamados seudoparadójicos. Esto quiere decir que aquellas paradojas que mediante un sesudo análisis revelen una falta de consecuencia lógica entre premisas y conclusión serán consideradas como falacias encubiertas. En términos quineanos, las paradojas falsídicas son pseudoparadojas. La propia palabra "pseudoparadoja" fue introducida por Stahl (1971, p. 94-96) en su opúsculo titulado "Al explorar lo infinito". Con esta palabra se alude a la calidad de un argumento cuya conclusión es falsa e inverosímil. Este conjunto de entes estará constituido por argumentos incorrectos que tendrán la apariencia de ser válidos, es decir, estará constituido por falacias, pero no por cualquier tipo de falacias sino solamente por aquellos malos argumentos considerados por la tradición como problemáticos. Toda pseudoparadoja es una falacia, pero no toda falacia es una pseudoparadoja, porque si bien hay un error de razonamiento, este necesita que el argumento sea reinterpretado (reformulado, corregido e interpretado) de manera un tanto diferente a la formulación original para lograr desestimar su aspecto contradictorio.

\section{La metafísica eleática}

Antes de entrar de lleno a la paradoja de Aquiles y la tortuga explicaremos un poco su contexto filosófico. Parménides de Elea, maestro de Zenón, sostuvo que la nada es impensable, y como tal no puede existir. Todo lo que es, simplemente, es. Y, como no podemos concebir el no-ser, es imposible que algo pueda nacer del no-ser. Intentemos pensar en la nada. Todo lo que se nos ocurra siempre será algo. Por eso, la nada es impensable, inconcebible, nada puede provenir del no-ser. Así, este pensador afirma que es necesario decir y pensar lo que es, pues es posible ser; mientras a la nada no le es posible ser, pues jamás se impondrá esto: que haya cosas que no sean. Recordemos su frase: "El ser es y el no-ser no es”. Además, Parménides sostenía que lo que percibimos como movimiento y cambio es solo mera ilusión. La realidad es una, permanente e indivisible. Es decir, el ser es inengendrado e imperecedero, íntegro, único en 
su género, incorruptible y plenamente realizado. Nunca fue ni será, puesto que es ahora, todo a la vez, uno y continuo. Para comprender esto pensemos en si el ser podría nacer o si podría morir. Decimos que no puede nacer. Pues si naciera significa que antes no era (no existía) y que después comenzó a ser. Pero, eso es imposible porque el ser siempre es y ha sido. Igualmente, el ser no puede morir, porque si muriera pasaría de ser al no-ser. Pero, eso es imposible porque el ser siempre es, ha sido y será. Así pues, se prueba que el ser no ha nacido ni ha muerto, es eterno.

Zenón de Elea encabezó la defensa de la filosofía de su maestro. Para sustentar la noción eleática del ser-uno elaboró una serie de paradojas que resaltaban el carácter contradictorio del movimiento. Para ello, Zenón asumía que tanto el espacio como el tiempo son infinitamente divisibles y, así, el movimiento es, en rigor, impensable. Asimismo, sostenía que si existe la multiplicidad, no se debe pensar que es necesario que sus integrantes sean tantos cuantos son, ni más que ellos ni menos, pues si fuesen tantos cuantos son, serían limitados. Más bien, si existe la multiplicidad, se debe considerar que los entes son ilimitados o infinitos, pues en medio de los entes siempre hay otros y nuevamente en medio de estos, otros más.

Asimismo, esto explica que, al aceptar que el movimiento sea un cambio de lugar en el tiempo, se originen contradicciones. Pues, si se parte de una representación del tiempo como una sucesión de momentos separados, entonces, al estudiar un objeto en movimiento en cada uno de los momentos de su traslado, se concluirá que, como permanece en reposo en cada uno de esos momentos, en suma, no se mueve (paradoja de la flecha). Y, si se considera el tiempo como un continuo infinito, entonces, dados dos móviles distanciados entre sí donde uno de ellos compite por alcanzar al otro, se concluirá que aunque la distancia entre ambos se va reduciendo, sin embargo, siempre seguiría existiendo y, por ello, nunca se encontrarían (paradoja de Aquiles y la tortuga).

\section{Paradoja de Aquiles y la Tortuga}

Según Aristóteles en la Física, al respecto de las paradojas eleáticas, aforma: "Cuatro son los argumentos de Zenón sobre el movimiento que crean dificultades a los que tratan de resolver los problemas que plantean" ${ }^{7}$. Escribe Nicola Abbagnano al respecto:

Pero los argumentos más famosos de Zenón son los que formuló contra el movimiento, que nos han sido conservados por Aristóteles. E1 primero es el llamado de la dicotomía: para ir de $A$ a $B$, un móvil tiene que efectuar primero la mitad del trayecto $A-B ; y$ antes aún, la mitad de esta mitad y así sucesivamente hasta el infinito; de tal manera que nunca llegará a B. El segundo argumento es el de Aquiles: Aquiles (o sea, el más veloz) nunca alcanzará a la tortuga (es decir, al más lento), 
pues la tortuga tiene un paso de ventaja. En efecto, antes de alcanzarla, Aquiles deberá alcanzar el punto de donde ha partido la tortuga de modo que ésta siempre tendrá ventaja. El tercer argumento es el de la flecha. La flecha, que aparece en movimiento, en realidad, está inmóvil: en efecto, en todo momento la flecha no puede ocupar sino un espacio igual a su largura y está inmóvil con respecto a este espacio; y como el tiempo está hecho de momentos, la flecha estará inmóvil durante todo el tiempo. El cuarto argumento es del estadio. Dos masas iguales, dotadas de velocidades iguales, deben recorrer espacios iguales en tiempos iguales. Pero si dos masas se mueven una contra otra desde las extremidades opuestas del estadio, cada una de ellas emplea en recorrer la longitud de la otra la mitad del tiempo que emplearía si una de ellas permaneciese quieta: de donde Zenón deducía la conclusión de que la mitad del tiempo es igual al doble. (Abbagnano, 1994, p. 34)

Como un caso particular de este tipo de razonamientos hagamos memoria de la paradoja de Aquiles y la Tortuga:

El segundo argumento es el llamado "Aquiles". Es éste: el corredor más lento no será nunca alcanzado por el más rápido, pues es necesario que el perseguidor llegue primero al lugar de donde partió el perseguido, de tal modo que el más lento estará siempre un poco más adelante (Barnes, 1992, p. 326)

Reescribe Borges su exposición:

Aquiles, símbolo de rapidez, tiene que alcanzar la tortuga, símbolo de morosidad. Aquiles corre diez veces más ligero que la tortuga y le da diez metros de ventaja. Aquiles corre esos diez metros, la tortuga corre uno; Aquiles corre ese metro, la tortuga corre un decímetro; Aquiles corre ese decímetro, la tortuga corre un centímetro; Aquiles corre ese centímetro, la tortuga un milímetro; Aquiles el milímetro, la tortuga un décimo de milímetro, y así infinitamente, de modo que Aquiles puede correr para siempre sin alcanzarla. Así la paradoja inmortal. (1966, p. 114)

La pseudoparadoja se presenta porque, por un lado, Aquiles no alcanzaría a la tortuga, y por el otro, sí. Aquiles no alcanzaría a la tortuga, porque después de haber corrido $10 \mathrm{~m}$, la tortuga ha avanzado $1 \mathrm{~m}$, después de haber corrido $1 \mathrm{~m}$, la tortuga habrá avanzado $10 \mathrm{~cm}$., después de haber corrido $10 \mathrm{~cm}$, la tortuga habrá avanzado $1 \mathrm{~cm}$, etc. Sin embargo, podemos también entender que la puede alcanzar sin dificultad ya que siempre que dos móviles distanciados tengan distinta velocidad y vayan en la misma dirección el más rápido alcanzará al más lento. Incluso se puede calcular que la debe alcanzar antes de los $12 \mathrm{~m}$, precisamente a los $11,111 \ldots \mathrm{m}$, es decir $11, \overline{1}$, o, de manera equivalente, $1010 / 9 \mathrm{~m}$; pero esta solución se lograría establecer mucho tiempo después. Por aquella época, Aristóteles fue uno de los primeros en buscarle respuesta a esta paradoja. 
La solución de Aristóteles incluyó un concepto de infinitud todavía vigente hasta el día de hoy. El infinito puede ser concebido de dos maneras en los términos aristotélicos de acto y potencia. En primer lugar, podemos hablar del infinito actual, es decir, aquella totalidad de elementos dados todos juntos de una vez. Este tipo de infinito se considera una entidad con identidad propia, una entidad en sí misma. Además, el infinito actual supone que en un mismo instante " $t$ " reunimos un número infinito de elementos existentes en simultáneo en ese instante. Ejemplos:

a) $\mathrm{B}=\{3,5,7,9, \ldots\}$ y $\mathrm{B}$ es infinito.

b) $\mathrm{C}=\{\mathrm{x} / \mathrm{x}$ es un punto de $\mathrm{AB}\}$ y $\overline{\mathrm{AB}}$ es una línea de infinitos puntos.

c) El número de números naturales es infinito, específicamente, $\aleph_{0}$.

En segundo lugar, podemos hablar del infinito potencial, es decir, de aquello que es ilimitado por contar con la posibilidad de crecer o decrecer indefinidamente una magnitud dada. De esta manera, Aristóteles afirma que lo infinito no es aquello más allá de lo cual no hay nada, sino aquello más allá de lo cual hay algo. En consecuencia, el infinito potencial supone que en cualquier instante " $\mathrm{t}$ " es posible seguir enumerando los elementos. Ejemplos:

a) Como hay infinitos primos, para todo primo hay siempre otro mayor.

b) El universo espacial es infinito. ${ }^{8}$

c) Si una línea es infinita, entonces puede prolongarse tanto como se necesite.

Ahora bien, para Aristóteles la infinitud existe, pero dicha infinitud no es un número infinito de algo, pues hablar de infinitud es hablar de una finitud que siempre puede expandirse. Según él, hay un número infinito de distancias entre este punto y la puerta, pero solo de un modo limitado. No importa cuán grande sea el número finito de distancias que se haya marcado mentalmente, $\mathrm{o}$, incluso, de manera física siempre se podrá marcar un número finito mayor. Esta finitud puede expandirse por siempre, pero seguirá siendo algo finito. Y, entonces, no debemos atravesar más que un número finito de distancias.

Siguiendo la senda de la solución aristotélica, los matemáticos del s. XIX buscaron una manera de resolver esta vieja paradoja. Escribe Michael Clark al respecto lo siguiente:

Hasta el siglo XIX no se encontró un procedimiento matemático satisfactorio para sumar estos intervalos. La solución consistió en definir la suma de una serie infinita como el límite al que converge la sucesión de sus sumas parciales. Por simplicidad, supongamos que los dos se mueven a velocidad constante y que Aquiles solo corre el doble de rápido que la tortuga, a la que ha concedido medio kilómetro de ventaja. (...) 
Cuando Aquiles recupera la ventaja que le había concedido a la tortuga, esta ha avanzado otro cuarto de kilómetro. Cuando Aquiles recorre este último tramo, la tortuga se encuentra un octavo de kilómetro por delante, y así sucesivamente. Los intervalos que recorre Aquiles son, expresados en fracciones de kilómetro,1/2, 1/4, 1/8, 1/16, ... Las sumas parciales son

\section{$1 / 2$ kilómetro}

$1 / 2+1 / 4$ de kilómetro $=3 / 4$ kilómetro

$1 / 2+1 / 4+1 / 8$ de kilómetro $=7 / 8$ de kilómetro

$1 / 2+1 / 4+1 / 8+1 / 16$ de kilómetro $=15 / 16$ de kilómetro,

y así sucesivamente.

La sucesión de sumas parciales será por tanto:

$1 / 2,3 / 4,7 / 8,15 / 16,31 / 31,63 / 64,127 / 128,255 / 256,511 / 512$, 1023/1024, 2047/2048, 4095/4096 ...

Esta sucesión continúa indefinidamente, acercándose cada vez más a 1. En este caso, el límite de la serie o la suma de los intervalos recorridos es 1 . Aquiles se aproxima gradualmente a la tortuga hasta que la alcanza. (Clark, 2009, p. 22-23)

Así, la pseudoparadoja se origina, porque en lugar de tomar la suma entera, se consideran los infinitos sumandos ${ }^{9}$. Aquiles no puede recorrer en un tiempo finito uno por uno los infinitos segmentos correspondientes a los sumandos. En cambio, puede recorrer perfectamente en un tiempo finito el segmento que corresponde a la suma entera, y para alcanzar a la tortuga hará exactamente eso. De esta manera, la paradoja se logra reducir a una estrategia para engañar: una falacia. Lo que podemos concluir es que existe la creencia gratuita de que la suma de infinitos sumandos, todos números reales, no puede tener un valor finito. Aquiles corre tranquilamente sin preocuparse de los infinitos segmentos en que Zenón le fraccionó su recorrido; él alcanzará a la tortuga a los 11, 1̄m, según la interpretación de Borgues. La oposición entre el pensamiento y la realidad, que Zenón trazó en este caso no existe; sólo apareció una oposición entre el pensamiento proto-científico y el cientifico, pues en esa época aún no se desarrollaban suficientemente las bases para el cálculo por aproximación de valores de series convergentes de infinitos términos. ${ }^{10}$

\section{Falacia del continuo}

Nuestra principal idea es la de concientizar a los interesados sobre nuestra denuncia que indica cierto parecido o analogía entre el "Aquiles" y la falacia del continuo. Por ello, lo que nos toca es tratar de definir el concepto de esta particular falacia. La mencionada argumentación inválida, también denominada como argumento de la continuidad o del montón, era llamada por los antiguos 
griegos como falacia del montón de trigo, de la barba y del calvo. Asimismo, incluso recibe el nombre de paradoja sorites, ya que "sorites" en griego quiere decir "pila, montón". Se le atribuye a Eubúlides de Mileto, filósofo griego de la escuela megárica. Esta consiste en asumir que pequeñas diferencias en una serie continua de sucesos son irrelevantes, o que posiciones extremas, conectadas por pequeñas diferencias intermedias, son la misma cosa porque no podemos establecer un límite objetivo para el cambio. (García Damborenea, 2000, p. 236) Pongamos dos ejemplos básicos:

-Dos granos no son un montón de trigo, tres granos tampoco, cuatro tampoco... Por ende, nunca habrá un montón mientras se añadan uno a uno.

-Si a alguien con cabello se le arranca un pelo, no queda calvo. Si se le vuelve a sacar otro pelo, tampoco queda calvo. Si se le saca un tercero pelo, sigue sin ser calvo. Y, así, pelo a pelo, nunca será calvo. ${ }^{11}$

Mostremos el primer ejemplo en términos formales:

-Si 2 granos no son un montón, entonces 3 granos no son un montón

-Si 3 granos no son un montón, entonces 4 granos no son un montón

-Si 4 granos no son un montón, entonces 5 granos no son un montón, etc.

Por lo tanto, si 2 granos no son un montón, entonces un número inmenso de granos no son un montón.

Estas falacias hacen uso de la vaguedad en las definiciones de los términos que contienen. Precisamente, aplicar el sentido común a conceptos vagos genera esta falacia o paradoja. 'Vaguedad' significa que aunque contamos con las características generales de un objeto tal, no podemos aplicar infaliblemente un predicado vago porque los límites no quedan claros. Pensemos en las siguientes preguntas: ¿Cuál es la altura máxima de un hombre de baja estatura? ¿Cuándo un óvulo fertilizado se convierten en una persona? ¿En que punto una cortina blanca llega a ser 100\% negra producto de la contaminación? ¿Cuántos años debe cumplir un niño para que le dejemos de llamar "niño"? ¿Cuánto dinero necesita un hombre para que lo llamemos "rico"? ¿Cuántos años necesita una persona para ser vieja? ¿Dónde comienza el exceso en la comida? ¿En qué punto la humildad se transforma en soberbia y el humor en burla? ¿Cuánta agua necesita el trigo para resplandecer? ¿Cuántos vellos se precisan para considerar que un hombre tiene barba? En el caso estudiado el término vago que ocasiona la paradoja es "montón".

\section{La relación entre el "Aquiles" y la falacia del continuo}

Ahora, reformularemos la paradoja de Zenón adecuadamente para que podamos constatar que su estructura es análoga a la mentada falacia: 
-En el primer instante, cuando Aquiles llegue a x, la tortuga estará en $\mathrm{x}+\alpha$ y aunque no la habrá alcanzado estará cerca de alcanzarla

-En el segundo instante, cuando Aquiles llegue a $\mathrm{x}+\alpha$, la tortuga estará en $x+\beta$ y aunque no la habrá alcanzado estará cerca de alcanzarla

-En el tercer instante, cuando Aquiles llegue a $x+\beta$, la tortuga estará en $x+\gamma$ y aunque no la habrá alcanzado estará cerca de alcanzarla, etc.

Por lo tanto, en el $\boldsymbol{n}$-ésimo instante, cuando Aquiles llegue a $\mathrm{x}+(\boldsymbol{n})$, la tortuga estará en $\mathrm{x}+(\boldsymbol{n}+\mathfrak{J})$ y aunque no la habrá alcanzado estará cerca de alcanzarla ${ }^{13}$

Por un lado, hemos mostrado que la pseudoparadoja zenónica se constituye como una violación de lo razonable, pues extiende y generaliza las distancias cada vez más cortas entre Aquiles y la tortuga afirmando que, de seguir así, nunca se tocarán. Siempre le faltará un poquito del recorrido y, por eso, nunca el pélida la alcanzará. Pero, matemáticamente, este asunto se desvanece porque, como hemos visto, la suma de infinitos elementos que van disminuyendo cuantitativamente puede dar lugar a una cantidad finita.

Por otro lado, en relación con la falacia del continuo, notamos cómo se puede asumir que pequeñas diferencias en una serie continua de sucesos son irrelevantes, dado que aunque siempre le faltará poquito para alcanzarla, puesto que la distancia que separa al corredor de la tortuga se acerca a cero, estrictamente nunca llegará a ser cero y, por ende, jamás se encontrará Aquiles con su inalcanzable tortuga (la cual a pesar de su lentitud característica llega a superar al griego físicamente más preparado de todos). El término vago que notamos en este caso se trata del constructo "y aunque no la habrá alcanzado estará cerca de alcanzarla” ¿Cuándo una cosa está cerca de alcanzar a otra? ¿Cuánta distancia debe separarlos para que está relación de "estar a punto de alcanzarlo" tenga sentido plenamente? ¿Deben estar a $1 \mathrm{~m}$ ? ¿a dos? ¿a la mitad? ¿a un cuarto de metro? ¿a 0, 000000000001 metro? Por lo tanto, lo que queríamos demostrar, a saber, que la paradoja de Aquiles es una falacia del continuo ha sido sustentada finalmente. Invitamos a juzgar a quienes muestren interés por este asunto los argumentos aquí vertidos. Mientras tanto podemos decir: “i L. Q. Q. D. !"

\section{Notas}

1 Pero no podemos decir que se dé una paradoja siempre que haya argumentos a favor de conclusiones incompatibles, pues, de lo contrario, todo asunto controvertido adquiriría carácter paradójico. Lo característico de tales paradojas es el hecho de que los argumentos divergentes son simétricos (es decir, se da tanto $\mathrm{p} \rightarrow \mathrm{q}$, como $\mathrm{q} \rightarrow \mathrm{p}$ ), lo que hace que la oposición resulte especialmente desconcertante.

2 Como aparece en el texto Elementos de Lógica de Óscar García (2012, p. 28-43). García Zárate procede a explicar el significado del término falacia siguiendo una estrategia de cuatro pasos: 
1) Distingue entre falacia y otros términos asociados con esta.

2) Indica el sentido coloquial del mismo y, enseguida, su sentido técnico.

3) Señala la importancia de su estudio.

4) Clasifica las mismas en base a ciertos criterios.

3 Un argumento es un tipo de discurso cuya estructura asocia dos elementos básicos:

1) El conjunto de las premisas (que pueden ser una o más de una)

2) El conjunto de la conclusión (que es única)

Un argumento puede ser, entre otras cosas, válido (cuando conserva la relación de consecuencia lógica) o inválido (cuando no la conserva).

4 En otras palabras, mientras que la falacia es un argumento en el que la relación de consecuencia lógica no tiene éxito, la paradoja es un argumento en el que, si bien hay consecuencia lógica, no obstante, exhibe inconsistencia, es decir, contradicción lógica.

5 La paradoja de Grelling registrada en el trabajo de Leonard Nelson y Kurt Grelling, "Bemerkungenzu den Paradoxien von Russell und Burali-Forti", y también en "Teoria de Conjuntos" del mismo Grelling (1943: 115-116) es también conocida como la 'paradoja de los términos heterológicos'. Según ella, muchas expresiones del lenguaje corriente pueden dividirse en autológicas y heterológicas. Expresiones autológicas son las que se refieren a sí mismas, esto es, expresiones de la forma: ' $t$ ' es $t$. Ejemplos de ellas en español son: 'breve' (que es breve); 'escrito en español' (que está escrito en español); 'impreso en negro' (que está impreso en negro); 'consta de cuatro palabras' (que consta de cuatro palabras). Expresiones heterológicas son las que no se refieren a sí mismas, esto es expresiones de la forma: ' $t$ ' no es $t$. Ejemplos de ellas son: 'escrito en francés' (que no está escrito en francés); 'impreso en rojo' (que no está impreso en rojo); 'consta de dos palabras' (que no consta de dos palabras). En medio de estas expresiones, el problema que se plantea es el siguiente: ¿El término 'heterológico' es heterológico o autológico?

6 La clásica paradoja de Bertrand Russell de la Teoría Lógica de las Clases fue descubierta por Zermelo en 1900 y un año más tarde, redescubierta independientemente, por Russell, quien la publicó. Se ha solido considerarla como una paradoja de la Teoría de Conjuntos debido a la confusión del concepto de conjunto con el concepto fregeano de clase (o extensión de un concepto). La fundamentación lógica de la Aritmética por Frege se basaba en el supuesto de que para cada propiedad o condición $\varphi(x)$, expresable en el lenguaje, existe la clase de todas las cosas que tienen esa propiedad o cumplen esa condición. Sucumbiendo a la confusión señalada, dicha clase se renresenta con el mismn símbolo que el conjunto de todas las x que cumplen la condición $\varphi(x)$, a saber, $\{x / \varphi(x)\}$. Naturalmente lns nhjetos de esta clase satisfacen la condición que la define, es decir, $\left(\nabla_{z}\right)(z \in\{x / \varphi(x)\} \leftrightarrow \varphi(z))$; donde alentamos la confusión utilizando el símbolo $\in$ para significar la pertenencia a una clase fregeana. Ciertamente hay clases que no se pertenecen a sí mismas, esto es clases $\mathrm{x}$ tales que $x \notin_{x}$; por ejemplo, la clase de todas las moscas no es una mosca. Russell se preguntó por la clase de todas las clases que cumplen esa condición, la clase $\mathrm{r}=\left\{x / x \notin_{x}\right\}$ de todas las clases que no se pertenecen a sí mismas. La suposición de que esta clase existe produce inmediatamente una contradicción. En efecto, $\left(\forall_{z}\right)\left(z \in\left\{x / x \notin_{x}\right\} \leftrightarrow z^{\oplus_{z}}\right)$.

7 Puede encontrarse una exposición adecuada y correcta en Mosterín y Torreti (2010, p. 457460).

8 Cito una noticia del 04-06-14 de Levante. "El Universo tal y como lo entendemos es realmente infinito en todos los sentidos, abierto y no cerrado, pero también es finito por 
el tiempo, porque es más grande que la distancia que ha recorrido la luz desde el "Big Bang" hasta hoy. De ahí que todo lo que esté fuera de esa distancia sea aún inaccesible”. Con este estilo pedagógico e instructivo, Alberto Fernández Soto, científico titular del Instituto de Física de Cantabria, respondió a la cuestión que todos nos hemos planteado de si el espacio es infinito o, por el contrario, tiene límites. Disponible en : http://www. levante-emv.com/comunitat-valenciana/2014/06/04/universo-infinito-espacio-finito-tiempo/1121030.html

9 E1 número $11,111 \ldots(11, \overline{1})$ puede tratarse, al igual que cualquier otro número real, como la suma de infinitos sumandos, en el caso de la interpretación de Borgues: 10; $1 ; 0,1 ; 0,01$; 0,$001 ; \ldots$

10 En línea con este argumento, la paradoja de Zenón es planteada como un acertijo o enigma resoluble con herramientas matemáticas que deshacen la supuesta anomalía con procedimientos sustentados y coherentes. Pero, en virtud de la honestidad académica, es necesario decir que algunos estudiosos mencionan que la paradoja referida no puede resolverse, porque la concepción de espacio que se maneja implícitamente, invita a entenderlo como algo infinitamente divisible. Esta visión implica que lo (material y) espacial tenga que ser una entidad semejante a las cantidades aludidas por los números, es decir, una cantidad que, por más pequeña que sea, siempre tendrá mitad. Obviamente, el tema queda abierto aunque según la mecánica cuántica es posible seguir dividiendo el micromundo de las partículas subatómicas aún más. Para Barnes, esta paradoja plantea ciertas cuestiones algo sabidas pero no explicitadas:

(...) El último paso del argumento no es válido. De:

(1) Para todo i, si Aquiles está en $\mathrm{C}_{\mathrm{i}}$, la tortuga estará en $\left[\mathrm{C}_{\mathrm{i}+1}\right]$ (sic),

Zenón nos invita a inferir:

(2) Para todo punto P de AB, si Aquiles está en p, la tortuga estará en un punto p', entre $\mathrm{p}$ y $\mathrm{B}$.

(1) es cierta; y así lo demuestra el argumento de Zenón. (2) no implica que Aquiles alcance a la tortuga. Pero (2) no se sigue de (1). (2) podría deducirse de la conjunción de (1) y:

(3) Para todo punto $\mathrm{p}$ de $\mathrm{AB}$, existe un i tal que $\mathrm{C}_{\mathrm{i}}$ se encuentra entre $\mathrm{p}$ y $\mathrm{B}$.

Pero es obvio que Zenón no puede contar con (3). Todo $\mathrm{C}_{\mathrm{i}}$ está entre $\mathrm{C}$ y el hipotético punto de encuentro $\mathrm{P}$; puesto que $\mathrm{C}_{\mathrm{i}}$ es el punto de $\mathrm{AB}$ en el que está la tortuga en el momento en el que Aquiles está en $\mathrm{C}_{\mathrm{i}-1}$. Así, con las dos suposiciones de que la tortuga nunca deja de moverse y que la velocidad de Aquiles es finita, no hay ningún i tal que $\mathrm{C}_{\mathrm{i}}=\mathrm{C}_{\mathrm{i}-1}$. Pero, si $\mathrm{C}_{\mathrm{i}}=\mathrm{P}$, entonces $\mathrm{C}_{\mathrm{i}}=\mathrm{C}_{\mathrm{i}-1}$, por tanto, no hay ningún i tal que $\mathrm{C}_{\mathrm{i}}=\mathrm{P}$. Evidentemente, ningún $\mathrm{C}_{\mathrm{i}}$ está entre $\mathrm{P}$ y $\mathrm{B}$. Por tanto, todos los $\mathrm{C}_{\mathrm{i}}$ estarán entre $\mathrm{C}$ y $\mathrm{P}$. Se sigue que Aquiles y la tortuga jamás se encuentran en un $\mathrm{C}_{\mathrm{i}}$, tal como se dice en (1). Pero se descubre [que] está conclusión es una trivialidad absoluta: los dos corredores nunca se encontrarán en ningún punto anterior a su primer punto de encuentro. De esto no se deduce nada como (2); del hecho de que no se encuentren antes de encontrarse apenas podemos inferir que nunca se encuentran. (1992, p. 327-328)

11 De acuerdo a Nancy Boyallian (2012) estos dos ejemplos muestran que la paradoja goza de la propiedad de reversibilidad dado que se mostraría una dirección ascendente y otra descendente: 
La más notoria peculiaridad de la paradoja de sorites, y quizás la prueba más evidente de su condición paradojal, es su reversibilidad. La misma consiste en la posibilidad de formular el argumento en sus ya conocidas dos direcciones.

La dirección ascendente:

(+) 'Un grano de arena no es un montón. E1 hecho de agregar un grano de arena a algo que no es un montón no lo convierte en un montón'. Si aplicamos un número considerable de veces estas premisas concluimos que 'no importa cuántos granos de arena agregue, nunca formaré un montón'.

Y la dirección descendente:

(-) 'Supongamos que estamos frente a un montón de arena. Si quito un grano de arena éste sigue siendo un montón'. Luego, la reiterada aplicación de estas premisas nos lleva a concluir que 'un simple grano de arena es un montón'. Conclusión que contradice la primera premisa de la dirección ascendente de la paradoja.

12 Estos ejemplos han sido sacados de Rodríguez (2013).

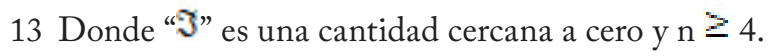

\section{Referencias bibliográficas}

Abbagnano, N. (1994) Historia de la Filosofía. Vol. 1, 4ta ed., Barcelona: Hora S. A.

Barnes, J. (1992) Los presocráticos. Madrid: Cátedra.

Blánquez Fraile, A. (1985) Diccionario Latino-Español. Barcelona: Editorial Ramón Sopena S. A.

Borges, J. L. (1966) Discusión. Buenos Aires: EMECÉ.

Boyallian, N. (2008) La paradoja del Montón. En XIV Jornadas de Epistemología de las Ciencias Económicas. 2 y 3 de octubre de 2008, en la UBA

Clark, M. (2009). El gran libro de las paradojas. Madrid: Gredos.

Copi, I. \& Cohen C. (2001). Introducción a la lógica. México: LIMUSA.

Dancy, J. \& Sosa, E. (comp.) (1992). A Companion to Epistemology. Massachussets: Blackwell.

De la Torre, A. (2000). Física Cuántica para filó-sofos. México: FCE.

Ferrater Mora, J. (1994) Diccionario de Filosofía. T. I-IV. Barcelona: Ariel.

Galilei, G. (1945). Diálogos acerca de dos nuevas ciencias. Buenos Aires: Losada.

García Damborenea, R. (2000) Uso de razón. Diccionario de falacias. Madrid: Biblioteca Nueva.

Grelling, K. (1943). Teoría de los Conjuntos. México: Logos de México.

Lopéz Arnal, S. \& De la Fuente, P. (2006) Paradojas: Con la ayuda del Juan de Mairena y textos afines. 29 junio, 2006. http://www.lainsignia.org/2006/junio/dial_009. htm. (1 agosto, 2007).

Łukowski, P. (2011). Paradoxes. University of Łódz: Springer. 
Mosterín, J. \&Torreti, R. (2010) Diccionario de Lógica y Filosofía de la Ciencia. Madrid: Alianza Editorial.

Pabón S. \& De Urbina, J. M. (1997) Diccionario Manual Griego-Español. Barcelona: VOX, (18 Edición).

Quine, W.V. (1976) "The Ways of Paradox". En: The Ways of Paradox and Other Essays, Cambridge-London, Harvard University Press, 1976, 1-18.

Rodríguez Cárdenas, F. (2013) "La lógica y la teoría epistémica: dos vías para abordar el problema de la vaguedad". En: Dialéctica, Año 2, No 2, pp. 205-214.

Scruton, R. (2003). Filosofía Moderna: una introducción sinóptica. 3era. edición, Santiago de Chile: Cuatro Vientos.

Stahl, G. (1971) Al explorar lo infinito. Santiago de Chile: Universitaria S. A. 